\title{
The Distribution and Feature Checking of Interrogative Sentences in Tao Te Ching
}

\author{
Ma Daoshan \\ Faculty of English Language, Tianjin Polytechnic University, Tianjin, People's Republic of China \\ Email address: \\ madaos@tom.com \\ To cite this article: \\ Ma Daoshan. The Distribution and Feature Checking of Interrogative Sentences in Tao Te Ching. International Journal of Language and \\ Linguistics. Vol. 4, No. 6, 2016, pp. 230-236. doi: 10.11648/j.ij11.20160406.15
}

Received: November 8, 2016; Accepted: December 5, 2016; Published: January 6, 2017

\begin{abstract}
An exhaustive description of interrogative sentences in the conventional version, the silk manuscripts and the bamboo slips of Tao Te Ching is done by qualitative approach. It is feasible to apply the Interrogative Feature Attraction Hypothesis to the explanation of the syntactic features of wh-questions in Tao Te Ching, and thus it is of great theoretical significance to the study of linguistic universality.
\end{abstract}

Keywords: Tao Te Ching, Interrogative Sentences, Syntactic Features

\section{Introduction}

Tao Te Ching, also called Laozi, a classic work before Qin Dynasty in ancient China, is a significant source of Taoist philosophy. The bamboo slips of Tao Te Ching [7] found in the Chu Dynastic Tomb at Guodian are written in three books of Book A, Book B and Book C. The silk scripts [6] are written in volume one and volume two. Volume one is called Te Ching and volume two is called Tao Ching originally, in which no chapters are subdivided. Later on Tao Ching is arranged in the first volume, and Te Ching in the second volume, in which 81 chapters are subdivided with about 5000 Chinese characters as a whole. The silk manuscript of Tao Te Ching is the earliest complete version of philosophical works in Chinese history. There are too many versions of Tao Te Ching, among which the traditional ancient classic version, the silk manuscript version and the bamboo slip version are the most popular. The bamboo slips of Tao Te Ching is the earliest ever existed version whose academic value has been well claimed, whereas the traditional ancient classic version is the most influential one with widespread popularity and the most numerous translated versions [5]. The most popular versions of Tao Te Ching in Chinese history are the Heshanggong version in Han Dynasty and the Wangbi version in Cao Wei Dynasty. Before Qing Dynasty, there had been more than 103 versions of Tao Te Ching, but the most valued versions in the academic circle are the Wangbi version and the two copies of silk manuscripts of Book $\mathrm{A}$ and Book B unearthed from Mawangdui, Changsha. The distribution of interrogative sentences in the bamboo slips. The silk manuscripts and the handed-down version of Tao Te Ching will be described respectively and the feature checking of interrogative features will be dealt with in the following sections in order to reveal the universaltiy of interrogation in Chinese.

There are 39 interrogative sentences in the handed-down traditional version of Tao Te Ching, among which 12 sentences are yes-no questions, up to $30.8 \%$ of the total,and 27 sentences are wh-questions, up to $69.2 \%$ of the total interrogative sentences in Tao Te Ching. There are 37 interrogative sentences in the silk manuscript of Tao Te Ching unearthed from the Han Dynasty Tomb in Mawangdui, among which 15 are yes-no questions, up to $40.5 \%$ of the total interrogative sentences in the silk manuscript, and 22 are wh-questions, up to $59.5 \%$ of the total. In the bamboo slips of Tao Te Ching, there are only 12 wh-questions.

In the following sections, the author will first describe the interrogative sentences in the bamboo slips, the silk manuscripts and the handed-down classic version of Tao Te Ching, and a syntactic explanation will be made based on the adequate description in Section 2 and Section 3.

\section{Yes-No Questions in Tao Te Ching}

\subsection{Yes-No Questions in the Traditional Handed-Down Version of Tao Te Ching}

In the traditional handed-down version of Tao Te 
Chingthere are altogether 12 yes-no questions, usually marked with the question particles of "hu"/ "ye"/ "yu" at the end of the sentences. These particles in archaic Chinese are used similarly in function with the modern Chinese question particle "ma", which all carry interrogative features and play a very important syntactic and semantic role in yes-no interpretation [11]. In fact, the modern Chinese grammarian tends to regard "hu"in archaic Chinese as the same functional question marker as the modern Chinese question particle "ma" used at the end of yes-no questions in modern Chinese [15].

There are 8 yes-no questions in the traditional handed-down version of Tao Te Ching ended with the interrogative particle "hu".

(1) Tiandi zhijian,qi youtuoyuehu?(Chapter 5)

- Universe in-between it like bellowsPART.

- "Inside the universe is it like the bellows?"

(2) Zaiying pobaoyi, neng wu lihu?(Chapter 10)

- Carry form soul embrace one, can no separation PART.

- "Can the form and soul carry life along never be separated from each other?"

(3) Zhuanqizhirou,neng ru yinger hu? (Chapter 10)

- Special Qi cause tenderness,can like baby PART.

- "Can the tenderness brought by special Qi be like a baby's?"

(4) Dichu xuanlan, neng wu ci hu? (Chapter 10)

- Cleanse inner-mind can no flawPART.

- "Can the inner mind be cleansed without any flaw?"

(5) Aiminzhi guo, neng wu wei hu? (Chapter 10)

- Love people govern country can no deed PART.

- "Can the love of the people and governance of the country be done without doing anything against the nature?"

(6) Tianmenkaihe, neng wei ci hu? (Chapter 10)

- Universe door open close can do female PART.

- "Can the door of the universe open and close naturally without being intentionally interfered?"

(7) Mingbaisida,neng wu zhi hu? (Chapter 10)

- Unequivocality mastery, can no knowledge PART.

- "Can the unequivocal mastery be done without any knowledge?"

(8) Fei hu? (Chapter 39)

- Non PART.

- "Is it non?"

There are 3 interrogative sentences in the traditional handed-down version of Tao Te Ching ended with the interrogative particle "ye".

(9) feiyiqiwusiye?(Chapter 7)

- Non with it no selfishness PART.

- "Isn't it without any selfishness?"

(10)cifei yijian wei ben ye?(Chapter 39)

- This non with base is basis PART.

- "Isn't this baseness regarded as the basis?"

(11)qiuyide,you zuiyimian ye ?(Chapter 62)

- Pursue with gain have sin with exempt PART.

- "Does it exempt sin by gaining it without any pursuit?"
Only 1 interrogative sentence is found in the traditional handed-down version of Tao Te Ching ended with the interrogative particle "yu".

(12)tianzhi dao, qi you zhanggong yu?(Chapter 77)

- Universe of law it like bending a bow PART.

- "Is the law of the universe like the bending of a bow?"

From the above examples, we can see that the yes-no questions in the traditional handed-down version of Tao Te Ching are marked with the question particles "hu", "ye" or "yu", these question particles carry interrogative force with them and can be applied to the interrogative reading of the sentences.

\subsection{Yes-No Questions in the Silk Manuscripts Version of Tao Te Ching}

The unearthed version of Tao Te Ching from the Han Dynasty Tomb in Mawangdui, Changsha is believed to be the silk manuscripts of Tao Te Ching in the Western Han Dynasty (206 B.C.-A.D. 24). In this silk version 8 yes-no questions are found to be ended with the question particle "hu" (the characters in [] are added in the blanks of the unearthed manuscripts), of which the first sentence is taken from the Te Ching and the others from Tao Ching.

(13)weiyou dao zhe hu?(Te Ching)

- Only have Dao zhe PART.

- "Is there the only Dao?"

(14)[Zaiying pobaoyi, neng wu li hu?](Tao Ching)

- Carry form soul embrace one, can no separation PART.

- "Can the form and soul carry life along never be separated from each other?"

(15)Zhuanqizhirou,neng ru ying'er hu? (Tao Ching)

- Special Qi cause tenderness, can like baby PART.

- "Can the tenderness brought by special Qi be like a baby's?"

(16)Xiuchu xuanlan, neng wu ci hu?(Tao Ching)

- Cleanse inner-mind can no flaw PART.

- "Can the inner mind be cleansed without any flaw?"

(17)Aiminhuo guo, neng wuyizhihu?(Tao Ching)

- Love people live country can no with knowledgePART.

- "Can the love of the people and governance of the country be done without any knowledge?"

(18)Tianmenqihe, neng wei ci hu? (Tao Ching)

- Universe door open close can do female PART.

- "Can the door of the universe open and close naturally without being intentionally interfered?"

(19)Mingbaisida, neng wuzyi wei hu? (Tao Ching)

- Unequivocal mastery, can no with deedPART.

- "Can the unequivocal mastery be like without doing anything against nature?"

(20)[you kuang]yurenhu? (Tao Ching)

- And morever about humanPART.

- "And what about the human beings?"

There are 2 yes-no questions ended with the question particle "ye" in the silk manuscripts of Tao Te Ching from Te 
Ching.

(21)Fei ye?

- Non PART.

- "Is it non?"

(22)qi wu zheng ye?

- It no uprightness PART.

- "Isn't it upright?"

There are 5 yes-no questions ended with the question particle "yu" in the silk manuscripts of Tao Te Ching.

(23)ciqi jian [zhi ben] yu?(Te Ching )

- This it base of basis PART.

- Is this the basis of baseness?

(24)youzui yimian yu?(Te Ching)

- have sin with exempt PART

- "Is the sin you have exempted?"

(25)feiyi qi wu zheng yu?(Te Ching )

- Non with it no struggle PART.

- "Isn't that he is fighting for it?" (Te Ching )

(26)[qi] you tuoyue yu?(Tao Ching)

- It have bellows PART.

- "Is it like the bellows?"

(27)buyiqi wu[si]yu? (Tao Ching)

- Not with it no selfishness PART.

- "Doesn't it carry no selfishness?"

From the examples quoted above, the yes-no questions in the silk manuscripts of Tao Te Ching is marked with question particles "hu", "ye" or "yu" at the end of the sentences, which carry interrogative features with them that play a very important role in the interpretation of the interrogation.

\section{Wh-questions in Tao Te Ching}

\subsection{Wh-questions in the Traditional Handed-Down Version of Tao Te Ching}

27 wh-questions are found in the handed-down classics of Tao Te Ching, of which wh-words are located in the initial position of 11 sentences, used as the subjects of the sentences, and different from yes-no questions there is no question particle in the sentences.

(28)heweiguidahuan ruo shen?(Chapter 13)

- What mean emphasize great illness like body

- "What does it mean by emphasizing great illness as if you have your body?"

(29)heweichongruiruojing?(Chapter 13)

- What mean favor humilation like surprise

- "What does it mean by nonsurprise at favor or humilation?"

(30)shu nengan yijiu?(Chapter 15)

- Who can quiet with long

- "Who can keep quiet for long?"

(31)shu neng zhuoyizhi?(Chapter 15)

- Who can chaos with stop

- "Who can stop the chaos?"

(32)shu wi ci zhe?(Chapter 23)

- Who do this PART.

- "Who does this?" (33)naihe yi wancheng zhi zhu, eryi shengqing tianxia? (Chapter 26)

- Why with allof Lord but with body lighten world

- "Why does the Lord of all take his body more precious than the world?"

(34)fuhegu?(Chapter 50)

- Oh what reason

- "What's the reason?"

(35)shuzhiciji?(Chapter 58)

- Who know this extreme

- "Who knows the extreme of this?"

(36)shu gan?(Chapter 74)

- Who dare

- "Who dares?"

(37)shu neng youyuyifeng tianxia?(Chapter 77)

- Who can have abundance to serve world

- "Who can serve the world with his abundance?"

There are 5 wh-questions in which the wh-words do not locate at the initial position of the sentences in the handed-down traditional classics of Tao TeChing, but at the end of the sentences, questions particles of "zai" or "hu" are actually used.

(38)wu heyizhizhongfuzhizhuang zai(Chapter 21)

- I what with know every creature of situation PART.

- "How do I know the situation of everything?"

(39)guzhi suoweiqu erquan zhe, qi xu yan zai?(Chapter 22)

- Ancient of so-called yield and merit PART. isn't empty talk P.

- "Isn't the so-called yielding merit empty talk in the ancient times?"

(40)tiandi shang bu neng jiu,er kuangyu renhu?(Chapter 23)

- Universe even not can long and morever in human PART.

- "Even the universe can't last long, to say anything of human beings?"

(41)wu heyizhitianxia ran zai? (Chapter 54)

- I what with know world this PART.

- "How do I know the universe like this?"

(42)wu heyizhiqi ran zai?(Chapter 57)

- I what with know it this PART.

- "How do I know it like this?"

In the handed-down classics we find 4 wh-questions with wh-words located at the end or in the middle of the sentences and with no question particles at the end. The elements located in front of the wh-words, in fact, can be considered as topics of the sentences.

(43)ming yu shen shu qin?(Chapter 44)

- Fame and body who dear

- "which is dearer, fame or body?"

(44)shen yu huo shu duo?(Chapter 44)

- Body and goods who more

- "Which is more, body or goods?"

(45)de yu wang shu bing?(Chapter 44)

- Gain and loss who harmful

- "Which is more harmful, gain or loss?" 
(46)guzhisuoyi guici dao zhe he?(Chapter 62)

- Ancient of why treasure this law PART. what

- "That's why ancient people treasure this law?"

7 wh-questions in the handed-down traditional classics of Tao TeChing carry relative clauses, in which no relative connectors are used, and therefore syntactically it is not obvious to read the sentences as relative clauses, but semantically the subordinate relationship between the two sentences can be interpreted, because Chinese is language which puts emphasis on concept instead of syntactic form.

(47)ji wu wu shen, wu you he huan?(Chapter 13)

- And I no body I have what illness

- "If I have no body, what illness do I have?"

(48)shanzhi yu e,xiangquruo he?(Chapter 20)

- Kindness it and evil difference like what

- "What is the difference between kindness and evil?"

(49)weizhi yu e,xiangqujihe?(Chapter 20)

- Respect it and vice difference how much

- "What is the difference between respect and vice?"

(50)renzhibushan, he qi zhi you?(Chapter 62)

- People it not kind what discard it have

- "What loss does it have if people are not kindhearted?"

(51)tianzhi suo e, shei zhi qi gu?(Chapter 73)

- Universe it of dislike who know its reason

- "Who knows the reason that it is dilliked by the universe?"

(52)minbuweisi,naihe yisiju zhi?(Chapter 74)

- People not frighten death how with death frighten them

- "If people are not afraid of death, how can you frighten them with death?"

(53)baoyuanyide, anhe yi wei shan?(Chapter 79)

- Treat complaints with virtue how with do good

- "If complaints are treated with virtue, how does good be done?"

From the syntactic features of wh-questions in the handed-down traditional classics of Tao TeChing, if the wh-words are located at the left periphery of the sentences, no questions particles are used at the end of the sentences, even in the (43)-(46) sentences that contain topics in left periphery. In wh-questions that carry relative clauses, no relative connectors are used.

\subsection{Wh-questions in the Silk Manuscripts Version of Tao Te Ching}

There are 22 wh-questions in the silk manuscripts of Tao TeChing, five of which carry the question particle "ye" at the end of the sentences. The following five wh-questions are quoted from Te Ching.

(54)fuheguye?

- Oh what reason PART.

- "Oh, what's the reason?"

(55)heguye?

- What reason PART.

- "What's the reason?"

(56)wu heyizhiqi ran ye?
- I what with know it this PART.

- "How do I know it's like this?"

(57)gu zhisuoyiguici zhe he ye?

- Ancient of why treasure this PART. what PART.

- "Why do ancient people treasure it like this?"

(58)naiheyishazhi ye?

- How with frighten it PART.

- "Why are you afraid of it?"

In the silk manuscripts of Tao TeChing, 1 wh-question carries the wh-question particle "zai' at the end of the sentence, quoted from Te Ching as follows:

(59)wu he yi zhi tianxia zhi ran zai?

- I what with know universe of this PART.

- "How do I know that the universe like this?"

Thereisalso1 wh-question from Te Chingthat carries the question particle "hu" at the end of the sentence:

(60)shuneng you yueryou yiqufengyu tian zhe hu?

- Who can have abundance and have with make offering to universe PART. PART.

- "Who can make offerings to the universe with the abundance of life he has?"

15 wh-questions don't carry any particles at the end of the sentences, which make up $68.2 \%$ of all the wh-questions in the silk manuscripts of Tao TeChing. (61)-(63) read exactly the same with (43)-(45).

(61)ming yu shen shu qin?(Te Ching)

- Fame and body who dear

- "Which is dearer, fame or body?"

(62)shen yu huo shu duo?(Te Ching)

- Body and goods who more

- "Which is more, body or goods?"

(63)de yu wang shu bing?(Te Ching)

- Gain and loss who harmful

- "Which is more harmful, gain or loss?"

(64)shu zhi qiji?(Te Ching)

- Who know its extreme

- "Who knows its extreme?"

(65)ren zhi bu shan ye, he qi zhi you?(Te Ching)

- People of no kindness Part. what loss it have

- "What loss does it have if people are not kind-hearted?"

(66)tian zhi suo e, shu zhi qi gu?(Te Ching)

- Universe of Passive disgust who know its reason

- "Who knows the reason why it is disgusted by the universe?"

(67)yan ke yi wei shan?(Te Ching)

- How can with do good

- "How can you do good with it?"

(68)-(75) are taken from Tao Ching.

(68)heweichongruiruojing?

- What mean favor humiliation like surprise

- "What does it mean by non-surprise at favor or humiliation?"

(69)heweiguidahuanruoshen?

- What mean emphasize great illness like body

- "What does it mean by emphasizing great illness as if you have your body?" 
(70)ji wu wu shen, you he huan?

- And I no body have what illness

- "If I have no body, what illness do I have?"

(71)weiyu e,qixiangqujihe?

- Respect and viceits difference how much

- "What is the difference between respect and vice?"

(72)meiyue,qi xiangqu he ruo?

- Beauty and ugliness its difference what like

- "What is the difference between beauty and ugliness?"

(73)wu he yi zhi zhongfu zhi ran?

- I what with know everything it this

- "How do I know everything like this?"

(74)shu wei ci?

- Who do this

- "Who does it?"

(75)ruohe yi wancheng zhi wang, eryi shengqing yu tianxia?

- Like what with allof Lord but with body lighten for world

- "Why does the Lord of all take his body more precious than the world?"

The above mentioned examples show that up to $68.2 \%$ of all the wh-questions in the silk manuscripts of Tao TeChing do not carry any wh-particle at the end of the sentences, while other wh-questions do carry some wh-question particles.

\subsection{Wh-questions in the Bamboo Slips Version of Tao Te Ching}

The bamboo slips of Tao Te Ching unearthed out of the tomb in Guodian, Jingmen, Hubei Province, is the earliest version that we have ever been able to read. In that version of Tao Te Ching altogether 12 interrogative sentences can be read, of which none is a yes-no question, and none carries any question particle at the end of the sentence.

(76)gudadao fei,an you renyi?(Bamboo Slips Book C)

- Ancient great law abandoned how exist benevolence

- "How can benevolence exist when the ancient great law is abandoned?"

(77)liuxinbuhe, an youxiaoci?(Bamboo Slips Book C)

- Kinship not harmonious how exist filial-piety

- "How can the filial piety exist without harmonious kinships?"

(78)bang jia hunluan, an you zheng chen?(Bamboo Slips Book C)

- State family dizzy how exist upright official

- "How can upright officials exist if the state and the family is dizzy?"

(79)weiyu e,xiangqujihe?(Bamboo Slips Book B)

- Respect and vicedifference how much

- "What is the difference between respect and vice?"

(80)meie,xiangqu he ruo?(Bamboo Slips Book B)

- Beauty ugliness difference what like

- "What is the difference between beauty and ugliness?"

(81)he wei chong rui?(Bamboo Slips Book B)

- What call favor humiliation
- "What is favor or humiliation?"

(82)heweiguidahuanruoshen?(Bamboo Slips Book B)

- What mean emphasize great illness like body

- "What does it mean by emphasizing great illness as if you have your body?"

(83)ji wu wu shen, huo he huan?(Bamboo Slips Book B)

- And I no body or what illness

- "If I have no body, what illness do I have?"

(84)wuhe yizhitianxiazhi ran?(Bamboo Slips Book B)

- I what with know universe of this

- "How do I know that the universe like this?"

(85)-(87) are quoted from the Bamboo Slips of Book $A$ which are exactly the same from (43)-(45) and (61)-(63):

(85)ming yu shen shu qin?

- Fame and body who dear

- "Which is dearer, fame or body?"

(86)shen yu huo shu duo?

- Body and goods who more

- "Which is more, body or goods?"

(87)de yu wang shu bing?

- Gain and loss who harmful

- "Which is more harmful, gain or loss?"

It seems that in the Warring Period, the Chinese wh-questions don't possess wh-particles at the end of the sentences. And what's more, wh-words in in the silk manuscripts of Tao TeChing canbe used as indefinites in declarative sentences, which are found in the Bamboo Slips of Book $A$.

(88)zhuneng zhuo yilaizhe, jiang qing.

- Who can chaos with come PART. will clear

- "Whoever can come from chaos will become clear."

(89)zhu neng ze yi wang zhe, jiang yu sheng.

- Who can worry with go PART. will rest life

- "Whoever can go out of worry will live for the rest of his life"

(90)wu keyi zhi qi ran ye.

- I could know it this PART.

- "I could know it like this."

\section{Feature Checking}

Chomsky [2] proposes that the motivation of movement is not greed principle but rather attraction. According to this proposal, Frampton [3] brought forward the Attraction Principle which says that syntactic movement is for the sake of satisfying the feature requirement of the head X [4]. The syntactic movement satisfies the need of the head instead of the need of itself. In light of the Attraction Principle, as a candidate to be attracted to the head $\mathrm{X}$, the feature of this phrase can move to the checking domain of the head $\mathrm{X}$ via movement operation to meet with the feature requirement of the head X. This movement operation can never occur unless the following two conditions are met: (1) There is no other candidate closer to the head X. (2) The formal features of the candidate match with those of the head X.

In German partial wh-movement constructions, the Spec position of $\mathrm{CP}$ in the matrix clause is always occupied by an 
interrogative domain marker, and the real wh-word is located in thespecifier position of the embedded clause [1]. Cheng [1] claims that the wh-feature moves to the $\mathrm{CP}$ position of the matrix clause, and then the wh-phrase partially pied-piped to the CP position in the embedded clause.

From the above analysis, the wh-feature first moves to the $\mathrm{CP}$ position of the embedded clause, and this triggers the pied-piping of the wh-phrase. And then the wh-feature moves to the $\mathrm{CP}$ position of the matrix clause, and this movement leaves the wh-phrase in the $\mathrm{CP}$ position of the embedded clause behind it Based on this hypothesis, Interrogative Feature Attraction Hypothesis [9], [10], [12], [13], [14] might be applied to the checking analysis of the syntactic features of interrogative sentences in Tao Te Ching in light of economy principle. As the interrogative sentences in Tao Te Ching are marked with question particles of "ye", "zai" and "hu" at the end of the sentences, and these question particles in archaic Chinese behave much similar with the question particle "ne" in modern Chinese, the just mentioned Interrogative Feature Attraction Hypothesis can be revised as follows:

(91)Interrogative Feature Attraction Hypothesis

In null specifier type of languages [8], the interrogative head with weak interrogative feature, located at the end of the interrogative sentence, which is represented as the question particle "ne"/ "ma" or the rising tone Q in modern Chinese, and "ye" / "zai" / "hu" in archaic Chinese, attracts the interrogative feature of the wh-word or the interrogative construction to move to spec $\mathrm{CP}$ position so that the interrogative feature is checked and thus the sentences can be interpreted as interrogative sentence.

The Interrogative Feature Attraction Hypothesis in (91) can be applied to the explanation of wh-questions in Tao Te Ching. As in the examples of (28)-(87), the wh-features of the wh-words "he" (what in English), "heyi" (why), "heru" (how), "jihe" (how much), "heye" (what), "shu" (who), "ruohe" (like what), "hekuang" (what's more), "an" (how) and "yan" (how/why) are moved respectively to the Spec position of CP in the initial position of the sentence to check the weak wh-feature of the head, and once checked, the sentence can be thus interpreted as wh-questions. The movement operation meet with the following 2 requirements of the Attraction Principle made by Frampton [3]: (1) There is no other candidate closer to the head X. (2) The formal features of the candidate match with those of the head X.. Let us take (38) as an example. (92) in figure 1 is the tree diagram of (38).

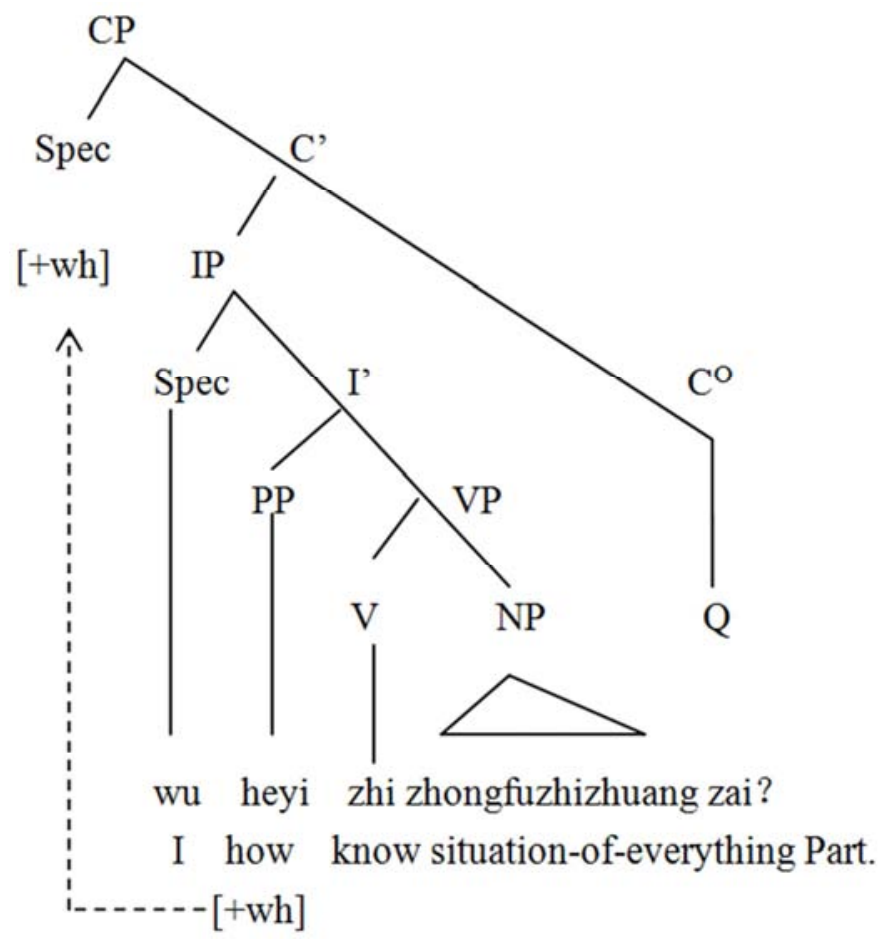

Figure 1. Tree diagram of (38).

As Chinese is a null specifier language [8], and the head feature in $\mathrm{C}$ of $\mathrm{CP}$ is weak, this weak head feature can not be checked off by attracting the whole category of the wh-word in (92) to move pied-piped to the spec position of CP. According to the Interrogative Feature Attraction Hypothesis in (91), the wh-feature carried by the wh-word is attracted to move to the spec position of CP to check off the weak head feature of the head $\mathrm{C}$, and after the feature is moved, the category of the wh-word remains in situ, and thus the sentence can be interpreted as a wh-question.

\section{Conclusion}

The author in this thesis has made an exhaustive description of the interrogative sentences in the handed-down classics of Tao Te Ching, the silk manuscripts of Tao Te Ching, and the 
bamboo slips of Tao Te Ching. Even though the syntactic and the semantic features of the interrogative sentences in the handed-down classics of Tao Te Ching, the silk manuscripts of Tao Te Ching, and the bamboo slips of Tao Te Ching are lightly different, the Interrogative Feature Attraction Hypothesis can be applied in the handed-down classics of Tao Te Ching, the silk manuscripts of Tao Te Ching, and the bamboo slips of Tao Te Ching to account for the syntactic feature checking operation of the interrogative sentences in the handed-down classics of Tao Te Ching, the silk manuscripts of Tao Te Ching, and the bamboo slips of Tao Te Ching. This shows that the hypothesis can be used to reveal the universal syntactic semantic features of interrogative sentences in natural languages.

\section{References}

[1] Cheng, Lisa Lai-Shen. Moving just the Feature[A]. In Uli Lutz, Gereon Müller, and Arnim von Stechow, eds. Wh-Scope Marking [C]. 2000: 77-99. Amsterdam: John Benjamins.

[2] Chomsky, Noam.The Minimalist Program [M]. Cambridge, Mass: The MITPress. 1995.

[3] Frampton, John. Expletive Insertion[A]. In Chris Wilder, Hans-Martin Gartner, and Manfred Bierwisch, eds., The Role of Economy in Linguistic Theory [C]. Berlin: Akademie Verlag. 1997.

[4] Stroik, Thomas. Locality in Minimalist Syntax [M]. Cambridge, Mass: The MIT Press. 2009.

[5] Chen, Guohua \& Xuan, Zhifeng.Versions of Laozi and the English Translation [J]. Foreign LanguageTeaching and Research, 2002 (6):464-470.
[6] Li, Er. The Silk Manuscripts of Laozi Unearthed in Mawangdui Tomb of Han Dynasty. [EB/OL]. http://blog.163.com... 2991929/,2009-12-29.

[7] Li, Er. The Bamboo Slips of Tao TeChing [EB/OL]. http://blog.163.com...2991929/, 2009-12-29.

[8] Ma, Daoshan. The Types of Natural Languages from the Perspective of Generative Studies of Wh-questions [J]. Contemporary Research in Modern Chinese, 2001, (3): $23 \sim 37$.

[9] Ma, Daoshan. The Syntactic Interrogation of the Particle "Ne" [Z]. (IACL-12) \& (ISCL-2), Tianjin: 2004-6-18-20.

[10] Ma, Daoshan.On the Syntactic Proof of the Interrogative Particle "Ne" [J]. Journal of Chinese Linguistics, 2006, (12):100-112.

[11] Ma, Daoshan. The Translation of Interrogative Sentences in Laozi and Its Typological Significance [J]. Theoretical Linguistic Studies, 2008, (2):101-108.

[12] Ma, Daoshan. A Syntactic Study of the Interrogative Sentences and Its Typological Significance in the Selected Readings from the Works of Mao Tsetung [J]. Journal of Beijing International Studies University, 2014(10): 14-23.

[13] Ma, Daoshan.The Syntactic Studies of Interrogative Sentences in the West and Their Defeciencies [M]. Guangzhou: World Book Publishing Co. Ltd., 2015.

[14] Ma, Daoshan. An Outline of English and Chinese Syntax [M]. Guangzhou: World Book Publishing Co. Ltd. 2016.

[15] Wang, Li. History of Chinese Grammar [M]. Beijing: The Commercial Press, 1989. 